\title{
0 impacto da incontinência urinária e seus fatores associados em idosas
}

\author{
The impact of urinary incontinence and their associated factors in elderly women
}

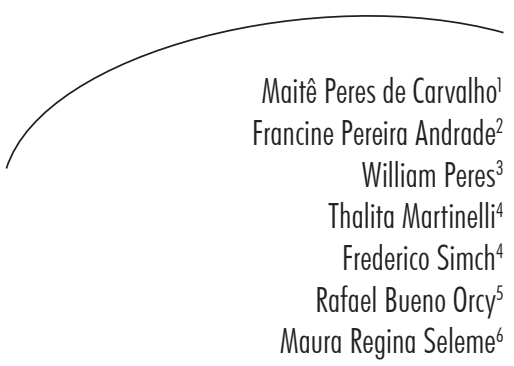

\section{Resumo}

Objetivo: Identificar a prevalência de incontinência urinária (IU) e fatores associados em idosas da comunidade. Métodos: Estudo transversal no qual foram entrevistadas idosas com 60 anos de idade ou mais que frequentavam um centro voltado exclusivamente a idosos em Pelotas-RS, Brasil. Foram utilizados como instrumentos desta pesquisa o International Consultation on Incontinence Questionnaire - Short Form (ICIQ-SF) e um questionário com informações complementares, que verificou fatores associados para a incontinência urinária. Além de estatística descritiva, foi utilizado o teste $t$ de Student para comparação das médias das variáveis quantitativas. Resultados: Foram avaliadas 132 idosas na faixa etária de 60 a 91 anos, com média de idade igual a 68,56 anos (dp $\pm 6,24)$. Em relação à perda de urina, a prevalência encontrada foi de 40,91\% (95\% IC $=32,6-49,2)$. Do total de mulheres analisadas, encontrou-se um índice de massa corporal (IMC) médio de $25,7 \mathrm{Kg} / \mathrm{m}^{2}$ (dp $\pm 4,06$ ), sendo que das idosas consideradas incontinentes foi demonstrado que $59,2 \%$ apresentavam IMC $\geq 27 \mathrm{Kg} / \mathrm{m}^{2}(\mathrm{p}<0.01)$, ou seja, com sobrepeso. Conclusões: A prevalência de IU encontrada (40,91\%) está dentro dos parâmetros registrados para esta faixa etária. Outro dado relevante é que o número de gestações aumentou a presença de IU, sendo que aquelas idosas que apresentaram três ou mais gestações foram proporcionalmente mais atingidas. O impacto da IU na qualidade de vida foi considerado ausente ou leve pela maioria das idosas, demonstrando que, provavelmente, a IU esteja em estágio inicial e não interfira de forma significativa no cotidiano dessas mulheres. Torna-se ainda relevante destacar que o diagnóstico precoce permite o tratamento adequado em tempo hábil, evitando maiores comprometimentos e melhorando a qualidade de vida.

\footnotetext{
Faculdade de Medicina, Programa de Pós-Graduação em Ciências da Saúde. Universidade Federal do Rio Grande. Rio Grande, RS, Brasil.

2 Faculdade de Enfermagem, Programa de Pós-Graduação em Enfermagem. Universidade Federal de Pelotas. Pelotas, RS, Brasil.

3 Centro de Ciências Químicas, Farmacêuticas e de Alimentos. Universidade Federal de Pelotas. Pelotas, RS, Brasil.

4 Centro de Ciências da Vida e da Saúde, Curso de Medicina. Universidade Católica de Pelotas. Pelotas, RS, Brasil.

5 Instituto de Biologia, Departamento de Fisiologia e Farmacologia. Universidade Federal de Pelotas. Pelotas, RS, Brasil.

6 Departamento de Pós-Graduação em Fisioterapia. Faculdade Inspirar. Curitiba, PR, Brasil.
}

Palavras-chave: Incontinência Urinária. Saúde da Mulher. Qualidade de Vida. Envelhecimento. Saúde Pública. 


\section{Abstract}

Objective: Identify the prevalence of urinary incontinence (UI) and associated factors in elderly women of the community. Methods: Cross-sectional study in which elderly women aged 60 or more who attended a center dedicated exclusively to elderly in Pelotas, Rio Grande do Sul state, Brazil, were interviewed. The instruments used were the International Consultation on Incontinence Questionnaire - Short Form (ICIQ$\mathrm{SF}$ ) and a questionnaire with additional information on associated factors for urinary incontinence. Besides descriptive statistics, Student's $t$ test was used to compare the means of quantitative variables. Results: The study evaluated 132 elderly women aged 60-91 years with mean age of 68.56 years (SD \pm 6.24 ). Regarding urine loss, the prevalence found was $40.91 \%$ (95\% IC $=32.6-49.2)$. Of all women interviewed it was found a body mass index (BMI) of $25.7 \mathrm{~kg} / \mathrm{m}^{2}(\mathrm{SD} \pm 4,06)$, and among elderly women considered incontinent, $59.2 \%$ had BMI $\geq 27 \mathrm{~kg} / \mathrm{m}^{2}$, that is, overweight. Conclusions: The prevalence of UI (40.91\%) found is within the registered parameters for this age group. Other relevant data is that the number of pregnancies increased UI, and elderly women with three or more pregnancies were proportionally more affected. The UI impact on the quality of life was considered absent or mild by most elderly women, probably showing that UI was in initial stage and did not interfere significantly in the everyday life of those women. It is still worth highlighting that early diagnosis allows appropriate treatment in a timely manner, avoiding major compromises and improving the quality of life.
Key words: Urinary Incontinence. Women's Health. Quality of Life. Aging. Public Health.

\section{INTRODUÇÃO}

De acordo com a International Continence Society (ICS), incontinência urinária (IU) é definida como uma condição na qual ocorre queixa de qualquer perda involuntária de urina, sendo um problema social ou higiênico muitas vezes erroneamente interpretado como parte natural do envelhecimento. Pode levar a um quadro clínico de depressão, isolamento e vergonha, alterando, portanto, o convívio social. ${ }^{1-4}$

Os três tipos mais comuns de IU são: de esforço (IUE), quando há perda involuntária de urina com esforços como ao tossir ou espirrar; de urgência (IUU), quando a paciente refere o desejo repentino de urinar e não obtém controle sobre o músculo detrusor; e mista (IUM), que é uma associação concomitante das duas formas supracitadas. ${ }^{5}$
A IU é multifatorial, mas algumas questões como idade avançada, multiparidade, cirurgias prévias e hipoestrogenismo, bem como deformidades pélvicas, contribuem para a perda da função esfincteriana. ${ }^{3}$ Além desses fatores, algumas alterações decorrentes do envelhecimento, como a atrofia dos músculos e tecidos, o comprometimento funcional do sistema nervoso e circulatório e a diminuição do volume vesical podem contribuir para o surgimento da IU, pois reduzem a elasticidade e a contratilidade da bexiga. ${ }^{5}$

Apesar de não ser uma condição de vida ameaçadora, a IU pode provocar diversos problemas de ordem física, como infecções, por exemplo, ou mesmo certo comprometimento psicossocial, afetando inclusive a qualidade de vida das mulheres caso não seja dispensada atenção adequada para o quadro clínico em tempo hábil. ${ }^{6-8}$ 
Há consenso na literatura demonstrando que a IU contribui para o surgimento de alterações psicológicas, de relacionamento pessoal, sexual e social, bem como de alterações físicas e econômicas, influenciando de forma negativa a qualidade de vida dessas pessoas. ${ }^{6,910}$ Também há registros de sintomas depressivos na literatura em mais de um terço das mulheres, cuja qualidade de vida se viu comprometida pelos sinais e sintomas da IU. ${ }^{11}$

Estudos internacionais apontam prevalência de $42 \%$ de IU entre mulheres ${ }^{12}$, aumentando para $44 \%$ naquelas com 65 anos ou mais. ${ }^{13}$ Portanto, sabe-se que o risco de desenvolver IU aumenta com a idade e que, aproximadamente, 50\% dos idosos institucionalizados apresentam IU, o dobro daqueles que vivem em comunidade. ${ }^{14}$

Doenças como depressão, acidente vascular cerebral, diabetes, e ainda, obesidade e limitações funcionais, aumentam consideravelmente as chances de sofrer IU. Em associação a fatores psicossociais e questões financeiras, a saúde física e a qualidade de vida dos idosos podem tornarse ainda mais deficitárias, assumindo proporção relevante para a saúde pública. Dessa forma, a adoção de medidas preventivas, diagnóstico precoce e tratamento adequado podem minimizar as consequências negativas da IU.15

Além disso, verifica-se que embora a IU seja um problema de saúde frequente entre os idosos, no Brasil são desenvolvidas escassas pesquisas, com amostra reduzida; assim, são necessários estudos com maior rigor metodológico que facilitem o planejamento de medidas adequadas de prevenção e tratamento, visando reduzir os gastos sanitários e diminuir o impacto dessa condição na saúde dos idosos. ${ }^{16}$

Com o intuito de conhecer mais detalhadamente tal problemática entre as mulheres idosas, objetivou-se identificar a prevalência de IU e fatores associados em idosas frequentadoras de um centro voltado à terceira idade.

\section{METODOLOGIA}

Trata-se de estudo de caráter transversal, no qual foram entrevistadas idosas com idade igual ou superior a 60 anos de idade que frequentavam o Centro de Extensão em Atenção à Terceira Idade (CETRES). Esse centro foi criado a partir de um projeto de extensão da Universidade Católica de Pelotas (UCPel), a fim de desenvolver ações educativas, socioculturais e de apoio à saúde mental, proporcionando maior qualidade de vida à comunidade idosa do município de Pelotas-RS.

O CETRES objetiva a socialização dos idosos, bem como o conhecimento e a atualização nos mais diversos campos; para tanto, oferece 21 oficinas em diferentes áreas, como dança, pintura em madeira, fisioterapia e estimulação da memória. Dessa forma, todas as idosas que frequentavam o centro no segundo semestre de 2010 e pertenciam à faixa etária estipulada foram convidadas a fazer parte do estudo, no total de 132 idosas. Era realizado um controle das idosas inscritas em cada oficina por meio de uma lista de presença fornecida pelo centro, de modo que, em caso de ausência em algum dia, a pesquisadora fez mais duas tentativas de entrevistar essa idosa. Não houve perdas ou recusas.

Utilizou-se como instrumento da pesquisa a versão completa em português do ICIQ-SF (International Consultation on Incontinence Questionnaire - Short Form), validado por Tamanini et al. ${ }^{17}$ Tratase de um questionário autoadministrável que avalia o impacto da IU na qualidade de vida e a qualificação da perda urinária dos pacientes analisados.

O ICIQ-SF é composto de quatro questões que avaliam a frequência, a gravidade (avaliada em duas questões distintas) e o impacto da IU, englobando um conjunto de oito itens de autodiagnóstico, relacionados às causas ou a situações de IU vivenciadas pelos pacientes, permitindo-os mencionar em que ocasiões há perda de urina. 
Também foi elaborado, pelos pesquisadores, um questionário com informações complementares, a fim de verificar fatores associados à IU. Tais questões permitiram delinear a média de idade da população, o nível de escolaridade e o índice de massa corporal (IMC), além do questionamento a respeito do número de gestações, idade da primeira gestação, número de partos normais, cesáreos e abortos.

O IMC foi obtido por meio do peso e da altura referidos pelas idosas. A classificação se deu através dos parâmetros preconizados pela Organização Mundial da Saúde ${ }^{18}$ para idosos, conforme segue:

$\begin{array}{cc}\text { IMC } & \text { Classificação } \\ <18,5 \mathrm{Kg} / \mathrm{m}^{2} & \text { Baixo-peso } \\ 18,5-24,9 \mathrm{Kg} / \mathrm{m}^{2} & \text { Normal } \\ \geq 25 \mathrm{Kg} / \mathrm{m}^{2} & \text { Sobrepeso } \\ 25,0-29,9 \mathrm{Kg} / \mathrm{m}^{2} & \text { Pré-obeso } \\ 30,0-34,9 \mathrm{Kg} / \mathrm{m}^{2} & \text { Obeso I } \\ 35,0-39,9 \mathrm{Kg} / \mathrm{m}^{2} & \text { Obeso II } \\ \geq 40,0 \mathrm{Kg} / \mathrm{m}^{2} & \text { Obeso III }\end{array}$

Para a análise dos dados, a amostra foi estratificada em dois grupos - continente e incontinente - para os quais se considerou, respectivamente, a ausência ou presença de qualquer queixa de perda involuntária de urina pela uretra. ${ }^{1}$

A análise do instrumento utilizado ${ }^{17}$ considera como incontinente a pessoa que relata pelo menos um episódio de perda urinária por semana durante os três meses anteriores à entrevista e, portanto, possui escore ICIQ-SF maior do que zero. Para tanto, é continente somente quem responde "nunca", "nenhuma" ou "não interfere" nas questões 3, 4, 5 e 6 do questionário.

A interferência da perda de urina na vida diária varia de 0 (não interfere) a 10 (interfere muito), sendo classificado em cinco categorias o impacto dessa interferência: (0) nada, (1-3) leve, (4-6) moderado, (7-0) grave e (10) muito grave. O escore ICIQ-SF é contabilizado pela somatória das questões 3, 4 e 5, oscilando de 0 a 21, em que quanto maior esse escore, maiores a severidade da perda urinária e o impacto na qualidade de vida. ${ }^{19}$

Os tipos de IU analisados a partir dos dados da questão 6 foram: IUE, quando há perda involuntária de urina com esforços; IUU, quando há o desejo repentino de urinar e não se obtém controle sobre o músculo detrusor; e IUM, que é uma associação da IUE e IUU.

Foi considerado com IUU quem respondeu pelo menos uma dessas alternativas: "perco antes de chegar ao banheiro"; "perco quando estou dormindo"; "perco quando terminei de urinar e estou me vestindo"; "perco sem razão óbvia"; "perco o tempo todo". Foi considerado com IUE quem respondeu: "perco quando tusso ou espirro" ou "perco quando estou fazendo atividades físicas". Para IUM, foi considerado quem respondeu pelo menos uma das opções de IUU e de IUE.

Para fins de cálculo do tamanho da amostra, utilizou-se o nível de confiança de $95 \%$ com a prevalência de IU de $56,85 \%,{ }^{20}$ obtendo-se um " $\mathrm{n}$ " de 95 idosas aceitando o máximo de diferença de $10 \%$. Os dados coletados foram duplamente digitados numa estrutura programada, por meio do programa EPIDATA versão 3.1. Para a análise dos dados, utilizou-se o software SPSS versão 17.0. Além de estatística descritiva, foi utilizado o teste $t$ Student para comparação das médias das variáveis quantitativas.

Como medida da consistência interna, foi aplicado o coeficiente Alfa de Cronbach, o qual é utilizado a fim de verificar a homogeneidade dos itens - ou seja, sua acurácia. Esse coeficiente verifica a correlação entre respostas em um questionário por meio da análise das respostas dadas pelos respondentes, apresentando correlação média entre as perguntas. Dessa forma, é possível verificar a confiabilidade de um teste em estudos transversais, em que o entrevistador só terá uma oportunidade de questionar o indivíduo. Como regra geral, a acurácia não deve ser inferior a 0,80 se a escala 
for amplamente utilizada, mas valores acima de 0,60 já indicam consistência entre os itens.

Este trabalho foi aprovado pelo Comitê de Ética em Pesquisa envolvendo seres humanos do Colégio Brasileiro de Estudos Sistêmicos, sob parecer $n^{\circ} 428 / 2010$, respeitando a Resolução n ${ }^{\circ}$ 196/96 do Conselho Nacional de Saúde (CNS). ${ }^{21}$ Todas as idosas que aceitaram participar do estudo assinaram o Termo de Consentimento Livre e Esclarecido.

\section{RESULTADOS}

Foram avaliadas 132 idosas na faixa etária de 60 a 91 anos, com média de idade igual a 68,56 anos (dp $\pm 6,24)$, sendo $54(40,91 \%)$ no grupo incontinente e 78 (59,09\%) no grupo continente.

Referindo-se ao grau de escolaridade das participantes, pode-se observar sua distribuição na tabela 1.

Tabela 1. Porcentagem de idosas de acordo com o grau de escolaridade e presença ou não de incontinência urinária. Pelotas-RS, 2011.

\begin{tabular}{cccc}
\hline Grau de escolaridade & $\%$ & Com IU & Sem IU \\
\hline Fundamental incompleto e completo & $32,5 \%$ & $35,1 \%$ & $30,7 \%$ \\
Médio incompleto e completo & $43,1 \%$ & $44,4 \%$ & $42,3 \%$ \\
Superior incompleto, completo ou pós-graduação & $24,2 \%$ & $20,3 \%$ & $26,9 \%$ \\
\hline
\end{tabular}

Na correlação de Pearson, a interferência da perda urinária na vida diária obteve associação de 0,67 com a frequência de perda de urina $(\mathrm{p}<0,0001)$; de 0,69 com a quantidade de perda urinária $(\mathrm{p}<0,0001)$; e de 0,33 com o IMC $(\mathrm{p}<0,0001)$ - apresentando, portanto, associação moderada. A associação entre o impacto na qualidade de vida e as variáveis "frequência de perda urinária", "quantidade dessa perda" e "interferência na vida diária" apresentaram associação forte, ou seja, maior que 0,7 ; enquanto o IMC apresentou associação moderada (tabela 2).

Quanto à perda de urina, a prevalência encontrada foi de 40,91\% (95\% IC $=32,6$ - 49,2), sendo que a maioria das incontinentes relatou perder urina uma vez por semana ou menos. (tabela 3).

Tabela 2. Associação entre o impacto na qualidade de vida e as variáveis: frequência de perda urinária, quantidade de perda urinária, interferência na vida diária e IMC. Pelotas-RS, 2011.

\begin{tabular}{lcccc}
\hline & $\begin{array}{c}\text { Frequência } \\
\text { de perda urinária }\end{array}$ & $\begin{array}{c}\text { Quantidade } \\
\text { de perda urinária }\end{array}$ & $\begin{array}{c}\text { Interferência } \\
\text { na Vida Diária }\end{array}$ & IMC \\
\hline $\begin{array}{l}\text { Impacto na qualidade de vida } \\
\text { (ESCORE ICIQ-SF) }\end{array}$ & 0,88 & 0,90 & 0,92 & 0,32 \\
& $\mathrm{p}<0,0001$ & $\mathrm{p}<0,0001$ & $\mathrm{p}<0,0001$ & $\mathrm{p}<0,0001$ \\
\hline
\end{tabular}


Tabela 3. Frequência que as idosas perdem urina. Pelotas-RS, 2011.

\begin{tabular}{lcc}
\hline \multicolumn{1}{c}{ Frequência com que perde urina } & $\mathrm{n}$ & $\%$ \\
\hline Nunca perdem urina & 78 & $59,1 \%$ \\
Uma vez por semana ou menos & 31 & $23,5 \%$ \\
Duas a três vezes por semana & 8 & $6,1 \%$ \\
Diversas vezes ao dia & 6 & $4,5 \%$ \\
Todo o tempo & 6 & $4,5 \%$ \\
Uma vez ao dia & 3 & $2,3 \%$ \\
\hline
\end{tabular}

Levando-se em consideração a interferência da perda de urina na vida diária, $46,2 \%$ das 54 incontinentes referiram que tal problemática não interfere no seu cotidiano.

Quanto ao impacto que a perda de urina gera em suas vidas, as idosas foram classificadas em cinco categorias: sem impacto $(77,9 \%)$, impacto leve $(7,6 \%)$, impacto moderado $(6,1 \%)$, impacto grave $(4,6 \%)$ e impacto muito grave $(3,8 \%)$. A média desse escore foi de 7,61 (dp $\pm 5,49)$.

O Alfa de Cronbach foi de 0,85 , demonstrando que o instrumento foi sensível e mensurou efetivamente o impacto da IU na qualidade de vida das idosas.

Caracterizando as causas ou situações de IU vivenciadas pelas idosas, encontrou-se a seguinte distribuição: IUE 33,3\% ( $\mathrm{n}=18)$; IUU 27,7\% $(\mathrm{n}=15)$; e em $38,8 \%(\mathrm{n}=21)$ dos casos verificouse IUM.

Do total de mulheres analisadas ( $\mathrm{n}=132$ ), encontrou-se um índice de massa corporal (IMC) médio de $25,7 \mathrm{Kg} / \mathrm{m}^{2}(\mathrm{dp} \pm 4,06)$. Houve diferença significativa entre as médias do grupo continente e incontinente (figura 1).

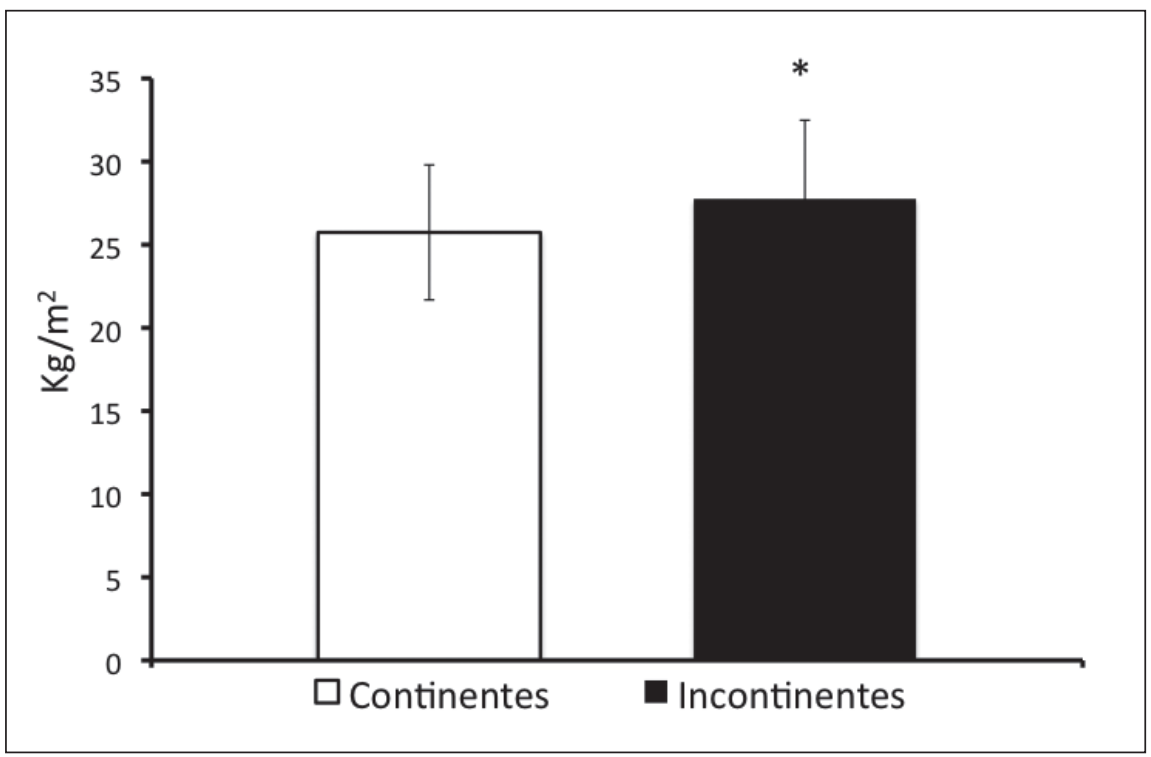

* p $>0,01$, Teste $t$ de Student.

Figura 1. Comparação entre as médias do IMC das pacientes. Pelotas-RS, 2011. 
Neste estudo, 117 mulheres (88,6\%) tiveram pelo menos uma gestação e, destas, $67,4 \%$ tiveram pelo menos um parto normal. A média de gestações foi de 2,51 ( $\mathrm{dp} \pm 1,38)$, enquanto a média de idade da primeira gestação era de 23,8 anos ( $\mathrm{dp} \pm 5,32)$. A média de partos normais foi de 1,75 (dp $\pm 1,52)$; cesáreos, 0,54 (dp $\pm 0,90)$; e os abortos foram 0,33 (dp $\pm 0,47)$. A associação dessas variáveis com as demais do estudo foi fraca.

Dentre as idosas com incontinência urinária, $51,85 \%$ tiveram três ou mais gestações, enquanto $25,93 \%$ relataram duas gestações; $2,97 \%$, apenas uma gestação e 9,25\% das entrevistadas eram nulíparas. As variáveis "escolaridade", "número de gestações" e "partos cesáreos" obtiveram associação fraca com as outras variáveis.

\section{DISCUSSÃO}

Neste estudo, a totalidade de idosas avaliadas obteve média de idade de 68,56 anos ( $\mathrm{dp} \pm 6,24)$ sendo que, quando comparadas as médias dos grupos continentes e incontinente, não houve diferença estatística. Outro estudo, ao verificar a ocorrência de IU aplicando o mesmo instrumento do referido estudo em idosos fragilizados atendidos no Ambulatório de Geriatria do Hospital de Clínicas da Universidade Estadual de Campinas, encontrou média de idade ainda mais elevada $\left(76,6\right.$ anos). ${ }^{22}$

Considerando os achados da literatura, a prevalência de IU eleva-se proporcionalmente em conjunto com a idade, sendo o principal fator de risco para a perda de urina. ${ }^{23-25}$ Pesquisas acerca do impacto na qualidade de vida nessas faixas etárias mais elevadas são fundamentais na tentativa de intervir especificamente em idosos, desmistificando a ideia de que IU é uma consequência natural do processo de envelhecimento e propondo a possibilidade de prevenção e/ou tratamento.

Pesquisa cujo objetivo foi identificar a taxa de prevalência, fatores associados e de risco para IU e o impacto na qualidade de vida de mulheres que compareceram ao programa de prevenção do câncer oncológico em Jaú/SP identificou a escolaridade como fator de risco para IU. ${ }^{19}$ Neste estudo, também foi possível observar tal tendência, visto que os menores índices de IU foram encontrados naquelas idosas que referiram, pelo menos, possuir ensino superior mesmo que incompleto. Esse fato é plenamente compreensível, pois aquelas com maior grau de escolaridade possuem conhecimento sobre os riscos e cuidados mínimos que a saúde requer ou ainda sabem como e onde buscar orientações a respeito, evitando, dessa forma, maiores agravos à sua saúde.

Estudos demonstram que existe grande discrepância entre as taxas de prevalência de IU encontradas na literatura $(8,5-55 \%) \cdot{ }^{26,27} \mathrm{~A}$ divergência entre os números pode apresentarse devido a fatores culturais, características sociodemográficas da amostra, hábitos de vida ou mesmo aos instrumentos utilizados nas pesquisas. Este estudo encontrou 40,9\% das idosas incontinentes, indo ao encontro de estudos internacionais que revelam índices de $42 \%$ a $44 \%$ de IU. ${ }^{12,13}$

De acordo com a frequência de perda de urina, $11,3 \%$ das idosas referiram apresentar esta problemática diariamente, enquanto outro trabalho encontrou $16,9 \%$ na população urbana de Pouso Alegre-MG, ${ }^{10}$ dados que intensificam a necessidade de uma abordagem precoce e eficaz na temática de IU, focando a prevenção precocemente.

Quanto ao aspecto da quantidade de perda de urina, a maioria das idosas incontinentes relatou perder pequena quantidade, fato que corrobora outros dois estudos que utilizaram o mesmo instrumento de coleta. ${ }^{28,29}$ É pertinente observar que este dado se relaciona diretamente com a frequência da perda urinária, em que $23,5 \%$ das idosas deste estudo relataram a frequência de uma vez por semana ou menos, podendo indicar que se trata de perdas ocasionais. Contudo, sabe-se que pode ser muito relativa a percepção de cada idosa a respeito da mensuração da perda urinária. Essa é justamente a principal 
limitação deste estudo, visto que o instrumento utilizado é autoadministrável e permite distintas interpretações.

A interferência da perda de urina na vida diária foi classificada em cinco categorias, de acordo com o preconizado pela literatura $;{ }^{19}$ na maioria dos casos, não se verificou impacto na qualidade de vida (77,9\%). Estudo apontou que 17,8\% das mulheres que procuraram o Programa de Prevenção do Câncer Ginecológico em JaúSP relataram impacto muito grave da IU na qualidade de vida, enquanto o presente estudo verificou apenas 3,8\% das idosas entrevistadas declarando impacto da mesma gravidade no seu cotidiano. Acredita-se que, por se tratar de idosas ativas, com relativo nível de escolaridade e bom convívio social no centro em estudo, o grau de comprometimento da IU não se encontra em nível elevado, demonstrando, portanto, pouco ou nenhum impacto na qualidade de vida. Ademais, o estudo realizado em SP possui um viés, visto que em um hospital oncológico a tendência é que as pacientes tenham maior grau de comprometimento de patologias associadas à região genital.

Quanto à estratificação da IU, outros trabalhos encontraram dados semelhantes aos deste estudo, sendo que a maior prevalência foi de IUM, ${ }^{5,30}$ seguida de IUE e de IUU. ${ }^{11,31}$ Se analisarmos separadamente apenas IUE, veremos que nosso estudo encontra-se com valor intermediário $(33,3 \%)$ em relação ao que tem sido verificado na literatura, 21,4\% $0^{32}$ e 39,6\%. ${ }^{11}$ Diversas revisões apontam que a associação entre IU e IMC se faz pertinente, pois nas mulheres com alto IMC, temos aumento da pressão intra-abdominal, provocada pelo aumento de peso na região da cintura e do quadril, gerando assim pressão excessiva intravesical e modificando o mecanismo do trato urinário. ${ }^{33} \mathrm{O}$ estudo em pauta teve associação fraca entre IU e IMC, da mesma forma que estudo realizado em Dourados-MS, referindose no entanto, especificamente, à IUE. ${ }^{32}$
Estudo desenvolvido em Jaú-SP identificou dados semelhantes ao da presente pesquisa no que se refere à média de gestações $(2,51)$ e de partos normais $(1,75) .{ }^{19}$ Trata-se de uma questão cultural que envolve custos e ausência de certos recursos em saúde há alguns anos; para tanto, os partos cesáreos eram menos prevalentes que os normais. Hoje, porém, é possível detectar que o parto normal está relacionado com o aumento de casos de IU quando comparado ao cesáreo, mas o parto normal não deve ser visto como o causador da IU, apenas quando associado a certos traumas no assoalho pélvico. ${ }^{34}$

Devem-se considerar algumas limitações para este estudo, visto que idosos frequentadores de centros voltados à terceira idade representam apenas uma parte da população e tendem a possuir maior qualidade de vida e independência funcional, se comparados aos idosos institucionalizados ou mesmo aos idosos da comunidade em geral. Ademais, as mulheres normalmente possuem mais cuidados com a saúde do que os homens, independentemente da faixa etária. ${ }^{35}$ Outra limitação se refere ao fato de o instrumento utilizado ser autoadministrável, permitindo distintas interpretações por parte de cada respondente, de acordo com sua percepção, nível de escolaridade e compreensão a respeito do assunto, apesar de o ICIQ-SF ser validado no Brasil. ${ }^{17}$ A não realização da análise multivariada também pode ser considerada uma limitação deste estudo.

\section{CONCLUSÕES}

Com base nos resultados deste estudo, foi possível concluir que a prevalência de IU encontrada $(40,91 \%)$ está dentro dos parâmetros registrados para esta faixa etária, mas não podemos consentir que esses valores sejam considerados aceitáveis.

Outro dado relevante é que o número de gestações aumentou a presença de IU, sendo que aquelas idosas que apresentaram três ou 
mais gestações foram proporcionalmente mais atingidas. O impacto da IU na qualidade de vida foi considerado ausente ou leve pela maioria das idosas, demonstrando que, provavelmente, a IU esteja em estágio inicial e não interfira de forma significativa no cotidiano dessas mulheres.

Com essas evidências, percebemos que qualificar profissionais para atender ao crescente número de idosos é uma necessidade premente, assim como preparar as estruturas e os serviços de saúde. Dessa forma, acreditase na fundamental importância desta temática, podendo os resultados encontrados na pesquisa colaborar na gestão dos serviços públicos em relação às necessidades dessa população.

Torna-se ainda relevante destacar que o diagnóstico precoce permite o tratamento adequado em tempo hábil, evitando maiores comprometimentos e melhorando a qualidade de vida. Ademais, evitar o sedentarismo e a obesidade, controlar o ganho de peso durante a gestação e manter a musculatura do assoalho pélvico fortalecida são medidas preventivas bastante eficientes.

\section{REFERÊNCIAS}

1. International Continence Society. Recommendations of the International Scientific Committee: evaluation and treatment of urinary incontinence, Pelvic Organ Prolapse and Faecal Incontinence. $4^{\mathrm{a}}$ International Consultation on Incontinence; 2008; Paris. Paris, França: ICUD; 2009 [acesso em 10 jun 2014]. [aproximadamente 54 p.]. Disponível em: http:// www.ics.org/Publications/ICI_4/files-book/ recommendation.pdf

2. Lopes MHB, Higa R. Restrições causadas pela incontinência urinária à vida da mulher. Rev Esc Enferm USP 2006;40(1):34-41.

3. Reis RB, Cologna AJ, Martins ACP, Paschoalin EL, Tucci Junior S, Suaid HJ. Incontinência urinária no idoso. Acta Cir Bras 2003;18(supl 5):47-51.

4. Rohr G, Christensen K, Ulstrup K, Kragstrup J. Reproducibility and validity of simple questions to identify urinary incontinence in elderly women. Acta Obstet Gynecol Scand 2004;83(10):969-72.

5. Dedicação AC, Haddad M, Saldanha MES, Driusso P. Comparison of quality of life for different types of female urinary incontinence. Rev Bras Fisioter 2009;13(2):116-22.

6. Stach-Lempinen B, Sintonen H, Kujansuu E. The relationship between clinical parameters and healthrelated quality of life as measured by the 15D in incontinent women before and after treatment. Acta Obstet Gynecol Scand 2004;83(10):983-8.

7. Hajjar RR. Psychosocial impact of urinary incontinence in the elderly population. Clin Geriatr Med 2004;20(3):553-64.
8. Melo BES, Freitas BCR, Oliveira VRC, Menezes RL. Correlação entre sinais e sintomas de incontinência urinária e autoestima em idosas. Rev Bras Geriatr Gerontol 2012;15(1):41-50.

9. Gunnel A, Jan-Erik J, Örjan G, Kerstin N. Urinary incontinence: prevalence, impact on daily living and desire for treatment. Scand J Urol Nephrol 2004;38(2):125-30.

10. Santos CRS, Santos VLCG. Prevalence of urinary incontinence in a random sample of the urban population of Pouso Alegre, Minas Gerais, Brazil. Rev Latinoam Enferm 2010;18(5):903-10.

11. Knorst MR, Resende TL, Goldim JR. Perfil clínico, qualidade de vida e sintomas depressivos de mulheres com incontinência urinária atendidas em hospitalescola. Rev Bras Fisioter 2011;15(2):109-16.

12. Melville JL, Wagner LE, Fan MY, Katon WJ, Newton KM. Women's perceptions about the etiology of urinary incontinence. J Womens Health 2008;17(7):1093-8.

13. Mardon RE, Halim S, Pawlson LG, Haffer SC. Management of urinary incontinence in Medicare managed care beneficiaries: results from the 2004 Medicare Health Outcomes Survey. Arch Intern Med 2006;166(10):1128-33.

14. Lazari ICF, Lojudice DC, Marota AG. Avaliação da qualidade de vida de idosas com incontinência urinária: idosas institucionalizadas em uma instituição de longa permanência. Rev Bras Geriatr Gerontol 2009;12(1):103-12. 
15. Tamanini JTN, Lebrão ML, Duarte YAO, Santos JLF, Laurenty R. Analysis of the prevalence of and factors associated with urinary incontinence among elderly people in the Municipality of São Paulo, Brazil: SABE Study (Health, Wellbeing and Aging). Cad Saúde Pública 2009;25(8):1756-62.

16. Jerez-Roig J, Souza DLB, Lima KC. Incontinência urinária em idosos institucionalizados no Brasil: uma revisão integrativa. Rev Bras Geriatr Gerontol 2013;16(4):865-79.

17. Tamanini JTN, Dambros M, D'Ancona CAL, Palma PCR, Netto NR Jr. Validation of the "International Consultation on Incontinence Questionnaire - short form”. Rev Saúde Pública 2004;38(3)1-6.

18. World Health Organization. Obesity: preventing and managing the global epidemic: report of a WHO Consultation. Geneva: WHO; 2000. (WHO Obesity Technical Report Series, n. 284)

19. Tamanini JD, Tamanini MMM, Mauad LMQ, Auler AMBAP. Incontinência urinária: prevalência e fatores de risco em mulheres atendidas no Programa de Prevenção do Câncer Ginecológico. BEPA, Bol Epidemiol Paul 2006;34(3):17-23.

20. Borges PLC, Bretas RP, Azevedo SF, Barbosa JMM. Perfil dos idosos frequentadores de grupos de convivência em Belo Horizonte, Minas Gerais, Brasil Cad Saúde Pública 2008;24(12):2798-808.

21. BRASIL. Resolução 196, de 10 de outubro de 1996. Conselho Nacional de Saúde. Resoluções. Disponível em: http://conselho.saude.gov.br/web_comissoes/ conep/aquivos/resolucoes/resolucoes.htm

22. Silva VA, Souza KL, D’Elboux MJ. Incontinência urinária e os critérios de fragilidade em idosos em atendimento ambulatorial. Rev Esc Enferm USP 2011;45(3):672-8.

23. Botelho F, Silva C, Cruz F. Incontinência Urinária Feminina. Acta Urol 2007; 24(1):79-82.

24. Du Moulin MF, Hamers JPH, Ambergen AW, Halfens RJG. Urinary incontinence in older adults receiving home care diagnosis and strategies. Scand J Caring Sci 2009;23(2):222-30.

25. Liapis A, Bakas P, Liapi S, Sioutis D, Creatsas G. Epidemiology of female urinary incontinence in the
Greek population: EURIG study. Int Urogynecol J 2010;21(2):217-22.

26. Silva APM, Santos VLCG. Prevalência da incontinência urinária em adultos e idosos hospitalizados. Rev Esc Enferm USP 2005;39(1):36-45

27. Anger JT, Saigal C, Litwin M. The prevalence of urinary incontinence among community dwelling adult women: results from the National Health and Nutrition Examination Survey. J Urol 2006;175(2):601-4.

28. Sebben V, Tourinho Filho H. Incidência da incontinência urinária em participantes do Creati do município de Passo Fundo/RS. Rev Bras de Ciên do Envelh Hum 2008;5(2):101-9.

29. Silva L, Lopes MHBM. Incontinência urinária em mulheres: razões da não procura por tratamento. Rev Esc Enferm USP 2009;43(1):72-8.

30. Feldner-Junior PC, Bezerra LR, Girão MJB, Castro RA, Sartori MG, Baracat EC, et al. Valor da queixa clínica e exame físico no diagnóstico da incontinência urinária. Rev Bras Ginecol Obstet 2002;24(2):87-91.

31. Leroy LS, Lopes MHBM, Shimo AKK. A incontinência urinária em mulheres e os aspectos raciais: uma revisão de literatura. Texto \& Contexto Enferm 2012;21(3):692-701.

32. Gomes GV, Silva GD. Incontinência urinária de esforço em mulheres pertencentes ao Programa de Saúde da Família de Dourados (MS). Rev Assoc Med Bras 2010;56(6):649-54.

33. Higa R, Lopes MHBM, Reis MJ. Fatores de risco para incontinência urinária na mulher. Rev Esc Enferm USP 2008;42(1):187-92.

34. Oliveira E, Zuliani LMM, Ishicava J, Silva SV, Albuquerque SSR, Souza AMB, et al. Avaliação dos fatores relacionados à ocorrência de incontinência urinária feminina. Rev Assoc Med Bras 2010;56(6):688-90.

35. Gomes R, Nascimento EF, Araújo FC. Por que os homens buscam menos os serviços de saúde do que as mulheres? As explicações de homens com baixa escolaridade e homens com ensino superior. Cad Saúde Pública 2007;23(3):565-74. 